\title{
Branching Processes and Evolution at the Ends of a Food Chain
}

\author{
Guido Caldarelli and Claudio Tebaldi \\ I.N.F.M., Istituto Nazionale di Fisica della Materia, Sezione di Trieste, Italy \\ and S.I.S.S.A./I.S.A.S., V. Beirut 2-4, I-34013 Grignano di Trieste (TS), Italy \\ Attilio L. Stella \\ INFM-Dipartimento di Fisica e Sezione INFN, Università di Padova, I-35131 Padova, Italy
}

(Received 14 February 1996)

\begin{abstract}
In a critically self-organized model of punctuated equilibrium, boundaries determine peculiar scaling of the size distribution of evolutionary avalanches. This is derived by an inhomogeneous generalization of standard branching processes, extending previous mean field descriptions and yielding $\nu=1 / 2$ together with $\tau^{\prime}=7 / 4$, as distribution exponent of avalanches starting from species at the ends of a food chain. For the nearest neighbor chain one obtains numerically $\tau^{\prime}=1.25 \pm 0.01$, and $\tau_{\text {first }}^{\prime}=1.35 \pm 0.01$ for the first return times of activity, again distinct from bulk exponents. [S0031-9007(96)00474-7]
\end{abstract}

PACS numbers: 87.10.+e, 02.50.-r, 05.40.+j, 64.60.Lx

Branching processes (BP) occur in many fields of physics and biology, ranging from nuclear reactors to polymers and population dynamics [1]. Within the context of self-organized criticality (SOC), introduced by Bak, Tang, and Wiesenfeld [2], BP, or correlated versions of them, are expected to underlie the physics of many models, describing sandpiles [2-4], earthquakes [5], river networks [6], or species mutations [7]. By evolving long enough, these models self-organize in stationary critical states with long-range correlations in space and time, and with avalanches of activity occurring at all scales. Avalanches are often believed to be described in terms of critical BP in the mean field (MF) limit. In the present Letter we introduce and solve an inhomogeneous generalization of the standard BP. This allows us to determine peculiar scaling properties of BP at boundaries. In a unifying perspective, such properties provide a substantial extension of previous MF descriptions of SOC models.

Bak and Sneppen (BS) [7,8] introduced a SOC model describing an ecology of interacting species evolving by mutation and selection. This model provides an illustration of the mechanisms determining intermittency (punctuated equilibrium [9]) and scaling [10] in the evolutionary activity. Below we show that such intermittency and scaling have a richer structure than appreciated so far. Indeed, at the level of universal properties, it is possible to draw a clear cut distinction between evolutionary activities occurring in the "bulk" and at the "boundary" of an ecology. Bulk and boundary refer to different locations of a given species within the network of interactions with other species conditioning its evolution.

In a coarse grained, simplified description, BS associate to the $i$ th species of an ecology a single fitness parameter, $x_{i}\left(0<x_{i}<1\right) . x_{i}$ represents the ability of species $i$ to survive: the higher $x_{i}$, the higher the barrier to overcome in order to switch a mutation in the species. A genetic mutation changes the barrier of the species and modifies also the barriers of the other species interacting directly with it. This interaction should represent the fact that two species, e.g., take part in the same food chain. Sites of a lattice can be used to represent the species: in this case neighbor (n.n.) species can be assumed as directly related biologically, and thus interacting.

The dynamical evolution rules are as follows. Starting from an initial fitness landscape, the $i$ with lowest $x, i_{\min }$, is selected to undergo a mutation and its fitness $x_{i_{\min }}$ is modified into a new one, chosen at random. Because of the interaction, also some neighbors of $i_{\min }$ get modified $x$ 's, as an effect of the previous mutation. For a linear chain with n.n. interactions this implies that $x_{i_{\min }-1}$ and $x_{i_{\min }+1}$ are replaced by new randomly chosen $x$ 's. In a standard MF description, on the other hand, the notion of position is completely lost and one can, e.g., choose to replace the fitnesses of a certain number, $K-1$, of other species selected at random, besides $i_{\min }$. This random neighbor (r.n.) model is the only one for which a MF treatment of avalanches could be set up so far $[8,11]$. However, the lack of any meaning for distance in this MF is a quite strong limitation, to the extent that the very notion of SOC can be legitimately questioned [12].

Avalanches corresponds to sequences of mutations in which the minimal $x$ species is always found among those resulting from genetic changes in previous stages, starting from a given ancestor mutation with $x_{i_{\min }}=\lambda$. In the system the minimal $x$ value does not exceed $\lambda$ for the whole duration of the avalanche. The probability $P$ that an avalanche involves $s$ mutations is expected to vary asymptotically as $P(s) \propto s^{-\tau}$ in the SOC state, in which for all avalanches $\lambda$ attains the value $x_{c}$, the sharp threshold of the stationary $x$ distribution [8].

So far, in models like the n.n. chain, $\tau$ and similar exponents have always been discussed as bulk quantities 
$[12,13]$, i.e., considering statistics of avalanches starting everywhere within large, periodic systems. Compared to those in the bulk, a species at one end of an open chain (e.g., main predator, or basic level of microscopic life) has less species directly or indirectly connected to it. The paths through which dynamical correlations can propagate starting from an initial mutation on the boundary are also reduced. So, e.g., in semi-infinite geometry, boundary avalanches could be characterized by peculiar exponents, different from the bulk ones. Demonstrating boundary scaling in models like the BS one is a challenge, especially at the analytical level. Indeed, in the context of SOC with extremal dynamics, exact results are essentially limited to the above mentioned MF treatment $[11,14]$. Consideration of boundary effects or other inhomogeneities clearly requires a meaningful notion of distance. We achieve this within a novel MF description of the BS model with n.n. interactions, generalizing the standard BP studied in probability theory [1].

The main scaling result for the random neighbor MF model is $\tau=3 / 2[8,11]$. This $\tau$ is consistent with MF $\mathrm{BS}$ avalanche dynamics being equivalent to a BP. An avalanche can be identified with a tree, where nodes represent species mutating within the avalanche. From each node, as many branches depart as there are species undergoing genetic change directly due to a mutation taking place at that node. The same species can act as a node more than once within an avalanche. The complex structure of correlations of the BS model is simplified in MF by assuming that, at each node, well defined, independent probabilities exist for all branchings compatible with the dynamical rules. Avalanches are generation trees, whose distribution in number of generating individuals, $s$, is given by $P$. The existing MF approach clearly cannot address exponents for diverging lengths, as defined, e.g., in a Landau approach to standard criticality. We introduce a characteristic length within MF through boundaries breaking translation invariance and leading to a position dependence of the BP description. Standard BP theory deals with the discrete transform $\tilde{P}(z)=\sum_{s=1}^{\infty} P(s) z^{s}$, on which the scaling of $P(s)$ produces singular behavior of the form

$$
\tilde{P}(z)=1-c(1-z)^{\tau-1}+\text { 1.s.t. }
$$

for $z \rightarrow 1^{-}$. In Eq. (1) $c$ is a suitable positive constant and the last term on the right-hand side indicates regular or less singular terms. Without making reference to relative locations of the species along the chain, the standard BP assumes that well defined probabilities, $p_{i}(i=$
$0,1,2, \ldots, K)$, apply to the events in which a given species undergoing mutation triggers subsequent genetic changes, in the same avalanche, in $i$ species, possibly including itself. Independence of branchings leads to the validity of Watson's functional equation [1]

$$
\tilde{P}(z)={ }_{z} G(\tilde{P}(z)),
$$

with $G(y)=p_{0}+p_{1} y+p_{2} y^{2}+\cdots+p_{K} y^{K}$. Equation (2) imposes a constraint on the $p_{i}$ 's consistent with a singularity of the form (1). Such constraint reads $G^{\prime}(1)=$ 1 and automatically fixes $c=\sqrt{2 / G^{\prime \prime}(1)}$ and $\tau=3 / 2$ as the only compatible exponent [15]. This result for $\tau$ is largely universal with respect to different choices of the parameters $p_{i}$ and relies only on the analyticity of $G$. A natural choice is $p_{i}=\left(\begin{array}{c}K \\ i\end{array}\right) x_{c}^{i}\left(1-x_{c}\right)^{K-i}$. In the r.n. model $x_{c}=1 / K$ [8], implying satisfaction of $G^{\prime}(1)=1$. Replacing $x_{c}$ by $\lambda<x_{c}$ would amount to consider off-critical avalanches $\left[G^{\prime}(1)<1\right]$, with $x_{c}-$ $\lambda$ playing the role of a temperaturelike field. Let us consider now a semi-infinite sequence of species on a chain. To each species is associated an integer coordinate $j=0,1,2, \ldots$. In a n.n. model the presence of the boundary requires us to allow for a $j$ dependence of the avalanche size distribution; thus, $P_{j}(s)$ or $\tilde{P}_{j}(z)$ will describe avalanches starting at site $j$ along the chain. This situation can still be analyzed within what we call here inhomogeneous BP. Since, as a consequence of a mutation at $j \geq 1$, at most three species can be further involved in the avalanche ( $K=3$ for the n.n. case), probabilities $p_{0}, p_{1}, p_{2}$, and $p_{3}$ will describe the possible outcomes of such a mutation. For convenience, and consistently with the above expressions of the $p_{i}$ 's in terms of $x_{c}$, one can further assume that with probability $p_{0}$ no further mutation takes place in the avalanche; with probabilities $p_{1} / 3$ and $p_{2} / 3$ the avalanche propagates, respectively, in any one and any two of the species in the set $\{j-1, j, j+1\}$; finally, $p_{3}$ is the probability that the avalanche involves all three species. In the MF spirit it is also sensible to assume $j$ independence for the $p_{i}$ 's as long as $j \geq 1$. Of course, there should be different probabilities $p_{i}^{\prime}$ for $j=0$, where the boundary imposes $p_{3}^{\prime}=0$. A possible choice made below is to assign $p_{0}^{\prime}=p_{0}+$ $\frac{1}{3} p_{1}, p_{1}^{\prime}=\frac{2}{3} p_{1}+\frac{2}{3} p_{2}$, and $p_{2}^{\prime}=\frac{1}{3} p_{2}+p_{3}$ at $j=0$, again implying equivalence of $j=0$ and $j=1$ with respect to single branch outcomes.

With the above positions, Eq. (2) is replaced by a full hierarchy of equations:

$$
\begin{aligned}
\tilde{P}_{0}(z)= & z\left(p_{0}^{\prime}+\frac{p_{1}^{\prime}}{2}\left[\tilde{P}_{0}(z)+\tilde{P}_{1}(z)\right]+p_{2}^{\prime}\left[\tilde{P}_{0}(z) \tilde{P}_{1}(z)\right]\right), \quad \tilde{P}_{j}(z)=z\left(p_{0}+\frac{p_{1}}{3}\left[\tilde{P}_{j-1}(z)+\tilde{P}_{j}(z)+\tilde{P}_{j+1}(z)\right]\right. \\
& \left.+\frac{p_{2}}{3}\left[\tilde{P}_{j-1}(z) \tilde{P}_{j+1}(z)+\tilde{P}_{j}(z) \tilde{P}_{j+1}(z)+\tilde{P}_{j-1}(z) \tilde{P}_{j}(z)\right]+p_{3}\left[\tilde{P}_{j-1}(z) \tilde{P}_{j}(z) \tilde{P}_{j+1}(z)\right]\right), \quad j \geq 1
\end{aligned}
$$


$\tilde{P}_{j}(z)$ should converge to the bulk solution of Eq. (2), for $j$ approaching infinity. Thus, it is advantageous to adopt the following ansatz:

$$
\tilde{P}_{j}(z)=\tilde{P}(z)+\Delta(z) e^{-q(z) j}+\text { 1.s.t. },
$$

where $q$ is an inverse length and $\tilde{P}$ is the solution of Eq. (2). As shown below, the assumed $j$ independence of $\Delta$ and $q$ is consistent, as corrections to it would involve only subleading singular terms for $z \rightarrow 1^{-}$. By substituting Eq. (4) into Eqs. (3) one can deduce singular behaviors of $\tilde{P}_{0}$ and $q$. For $z \rightarrow 1^{-}$, we expect $\Delta(z) \sim$ $(1-z)^{\alpha}$ and $q(z) \sim(1-z)^{\beta}$, with $\alpha$ and $\beta$ suitable exponents. After substitution in Eqs. (3) for $j \geq 1$ one gets

$$
\begin{aligned}
1= & \frac{z}{3}[1+2 \cosh q(z)] \\
& \times\left(G^{\prime}(\tilde{P}(z))+\frac{\Delta(z)}{2} G^{\prime \prime}(\tilde{P}(z))\right)+\text { l.s.t. }
\end{aligned}
$$

Taking into account that $\tilde{P}$ has the form (1) with $\tau=$ $3 / 2$, the leading singular terms in Eq. (5) give

$$
\frac{q(z)^{2}}{3 G^{\prime \prime}(1)}+\frac{1}{2} \Delta(z)=a(1-z)^{1 / 2}+\text { 1.s.t. }
$$

where $a=c$ of Eq. (1). The same kind of substitution in the first of Eqs. (3) leads to

$$
\Delta(z)=a(1-z)^{1 / 2}-b \Delta(z) q(z)+1 . s . t .,
$$

with $b=1$. Equations (6) and (7) determine both $\alpha$ and $\beta$ above. In particular, $\tilde{P}_{0}(z)$ takes the form

$$
\begin{aligned}
\tilde{P}_{0}(z) & =\tilde{P}(z)+\Delta(z)+1 . \text { s.t. } \\
& =1-\sqrt{3 a} b(1-z)^{3 / 4}+1 \text {.s.t. }
\end{aligned}
$$

In general $b=\left(p_{1}^{\prime} / 2+p_{2}^{\prime}\right) /\left[2\left(p_{1}^{\prime} / 2+p_{2}^{\prime}\right)-1\right]$ and the results (6) and (8) make sense for $\sum_{i} i p_{i}^{\prime}<1$. This condition is satisfied by our choice of $p_{i}^{\prime}$, s, which further acquire the form $p_{i}^{\prime}=\left(\begin{array}{c}K_{i}-1 \\ i\end{array}\right) x_{c}^{i}\left(1-x_{c}\right)^{K-1-i}$, if the $p_{i}$ 's are expressed in terms of $x_{c}$ as discussed above. Thus, the threshold $x_{c}$ for the distribution of $x$ values at the borders in the stationary state should be the same as in the bulk. According to Eqs. (1) and (8)

$$
P_{0}(s) \sim s^{-7 / 4} .
$$

Thus, in our MF description the BS SOC state is characterized by a boundary scaling with an exponent $\tau^{\prime}=7 / 4$ different from the bulk one. Boundary avalanches of course suffer more rapid extinction and their distribution decreases faster for large $s$. It is interesting to note that, by exploiting analogies with magnetic systems, $\tau^{\prime}=7 / 4$ has been predicted recently within a MF approach to bor- der avalanches in Abelian sandpile models (ASM) with Dirichlet boundary conditions [16]. This lends further support to the idea that in ASM a BP description underlies the statistics of avalanches in the MF limit, for which also $\tau=3 / 2$ is expected [17]. By a numerical approach one can also identify $\tau^{\prime} \simeq 7 / 4$ for MF avalanches of the earthquake model of Refs. [5,18], confirming an underlying $\mathrm{BP}$ also in this case. A further consequence of Eqs. (6) and (7) is the singularity

$$
q(z) \sim(1-z)^{1 / 4} .
$$

Thus, the penetration length of the border disturbance, $q^{-1}$, diverges for $z \rightarrow 1^{-}$. In MF treatments of inhomogeneous equilibrium models, quantities like $q^{-1}$ show the same divergence with temperature as classical correlation lengths. By interpreting $z$ as a standard fugacity for a polymer, one deduces from Eq. (10) a correlation length exponent $\nu=1 / 4$. This is indeed the classical $\nu$ of branched polymers [19]. Of course the definition of $\nu$ for a SOC system requires one to identify physically meaningful parameters describing the approach or the departure from criticality. For BS avalanches such a parameter is the temperaturelike deviation $x_{c}-\lambda$. By introducing $\lambda$-dependent $p_{i}$ 's and $p_{i}^{\prime}$ 's in our equations, the result (10) can be converted into $q(\lambda, z=1) \sim\left(x_{c}-\right.$ $\lambda)^{1 / 2}$, which implies $\nu=1 / 2$. Remarkably enough, this is the classical $\nu$ exponent expected for ASM [17]. This and the above mentioned coincidence of $\tau^{\prime}$ strongly support the idea that BP fully underlie also the MF description of ASM avalanches.

In order to identify boundary scaling beyond MF, we performed systematic simulations with open, n.n. BS chains of different lengths $\left(N \leq 10^{3}\right)$. First we verified that the distribution of boundary $x$ 's in the stationary state is essentially unaltered with respect to that of the periodic, bulk case, and displays the same sharp threshold at $x_{c}=0.665 \pm 0.015$ [7]. This coincidence is fully consistent with our choices of the $p_{i}^{\prime}$ 's in the MF approach. By selectively sampling avalanches starting near the boundaries or in the interior of the chains, we extrapolate $\tau^{\prime}=1.25 \pm 0.01$ (see Fig. 1). This value is clearly different from the bulk one $\tau \simeq 1.08$ [12].

So, also in the n.n. model boundary avalanches have a probability of decaying more rapidly at large $s$, than in bulk. A further characterization of boundary scaling is given by the distribution of first return times of activity ( $x$ taking the minimum value) at the same boundary site. These times are distributed as $t^{-\tau_{\text {first }}^{\prime}}$, with $\tau_{\text {first }}^{\prime}=$ $1.35 \pm 0.01$, different from the bulk value $\tau_{\text {first }}=1.58$ [13]. By recording the times of all subsequent returns of activity one can also obtain a distribution $\propto t^{-\tau_{\text {all }}^{\prime}}$, with $\tau_{\mathrm{a} 11}^{\prime}=0.65 \pm 0.01$, again distinct from $\tau_{\mathrm{all}}=0.42$ in bulk [13]. Such boundary exponents are consistent with a scaling relation $\tau_{\text {first }}^{\prime}+\tau_{\text {a11 }}^{\prime}=2$, already satisfied in the bulk [13]. Since the validity of such a relation should 


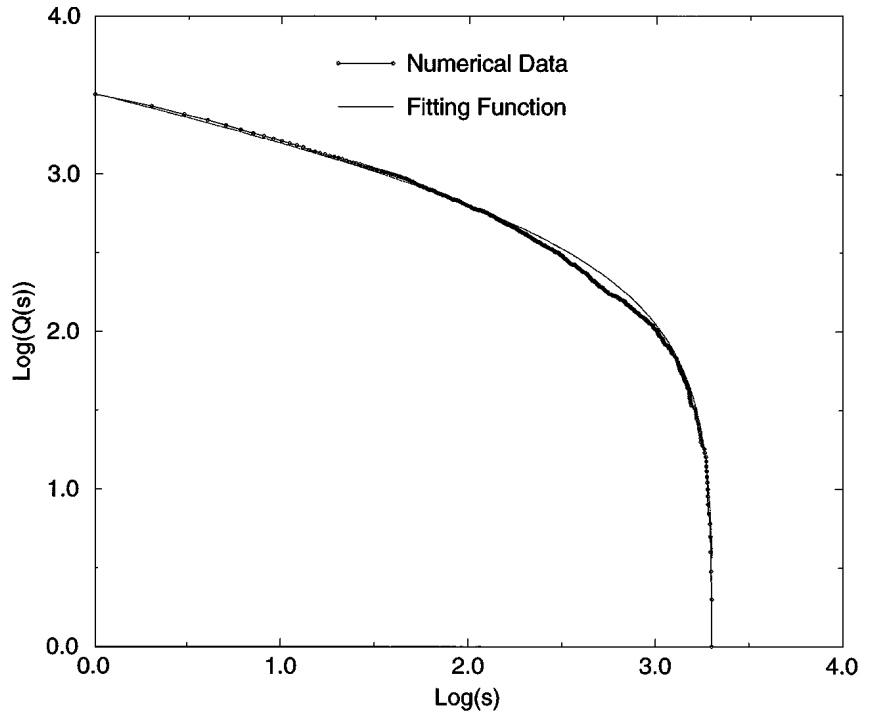

FIG. 1. $Q(s)=\int_{s}^{s_{\max }} P\left(s^{\prime}\right) d s^{\prime}$ is the integrated distribution; the fitting form is $A s^{1-\tau^{\prime}}+C$ with $\tau^{\prime}=1.25 \pm 0.01(N=$ 1000).

not depend on the position considered along the chain, the above consistency is further indication of the good quality of our determinations. Data concerning these exponents are shown in Fig. 2.

We conclude that at the boundaries activity has a different pattern of intermittency. First returns are shifted towards longer time scales. On the other hand, once the boundary has been reached, activity remains more easily trapped there, giving rise to concentrated sequences of returns. In applications of the BS model, the choice of a more or less regular network of interactions remains to some extent arbitrary, and should not matter for universal properties, unless the long-range limit of a r.n. model is

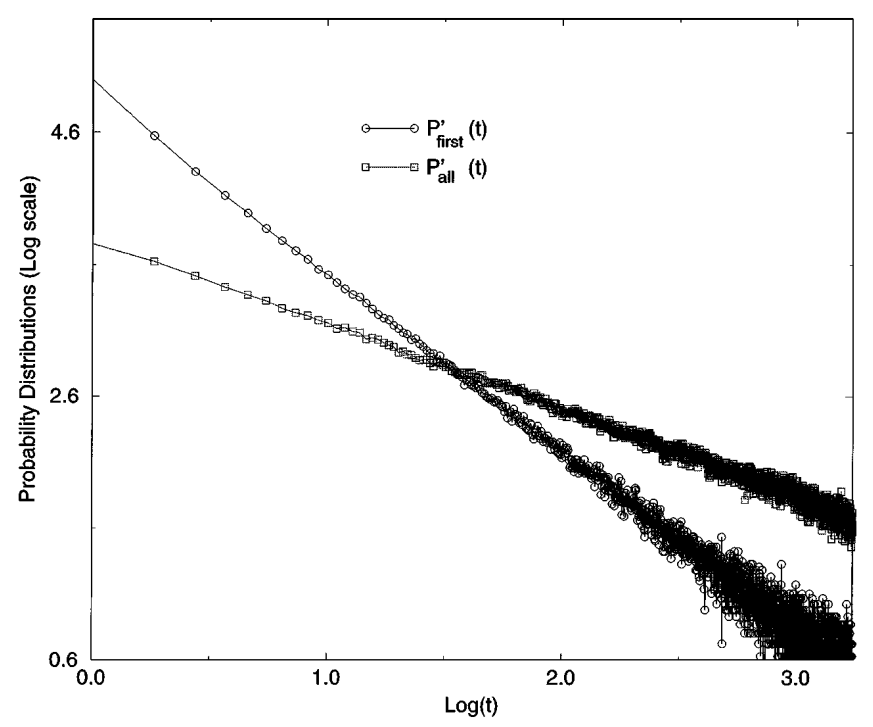

FIG. 2. First and all return times probabilities at boundary sites. Statistics refer to $10^{9}$ mutations in the whole chain. assumed. However, the distinction elucidated above between bulk and boundary species appears to have important consequences, affecting the universal scaling features of evolution. Thus, boundary scaling offers additional, deeper insight into the properties of biological models and widens the context of their possible comparison with paleontological data. Summarizing, we showed here that within the framework of punctuated equilibrium there exists a well defined boundary scaling in addition to the bulk one. At the MF level this scaling can be analyzed exactly within a generalization of BP theory, which considerably extends previous classical descriptions of the BS model, and directly focuses on its relation with other models. In particular also ASM with Dirichlet boundary conditions fall fully in the MF universality class of our BP. Also in the n.n. case our results show the existence of new scalings which make the notion of species at the ends of a chain meaningful in a universal sense.

[1] T. Harris, Theory of Branching Processes (Springer, Berlin, 1963); W. Feller, An Introduction to Probability Theory and its Applications (Wiley \& Sons, New York, 1971).

[2] P. Bak, C. Tang, and K. Wiesenfeld, Phys. Rev. Lett. 59, 381 (1987).

[3] P. Alstrøm, Phys. Rev. A 38, 4905 (1988); S. Zapperi, K. B. Lauritsen, and H.E. Stanley, Phys. Rev. Lett. 75, 4071 (1995).

[4] L. Pietronero, A. Vespignani, and S. Zapperi, Phys. Rev. Lett. 72, 1690 (1994).

[5] Z. Olami, H. J. S. Feder, and K. Christensen, Phys. Rev. Lett. 68, 1244 (1992); S. Lise and H. J. Jensen, Phys. Rev. Lett. 76, 2326 (1996).

[6] A. E. Scheidegger, Bull. Assoc. Sci. Hydrol. 12, 15 (1967); H. Takayasu, J. Stat. Phys. 65, 725 (1991).

[7] P. Bak and K. Sneppen, Phys. Rev. Lett. 71, 4083 (1993).

[8] H. Flyvbjerg, K. Sneppen, and P. Bak, Phys. Rev. Lett. 71, 4087 (1993).

[9] N. Eldredge and S. Gould, Nature (London) 332, 211 (1988).

[10] D. M. Raup, Science 231, 1528 (1986).

[11] J. de Boer, B. Derrida, H. Flyvberg, A. D. Jackson, and T. Wettig, Phys. Rev. Lett. 73, 906 (1994).

[12] J. de Boer, A. D. Jackson, and T. Wettig, Phys. Rev. E 51, 1059 (1995).

[13] S. Maslow, M. Paczuski, and P. Bak, Phys. Rev. Lett. 73, 2162 (1994).

[14] As an exception, see S. Boettcher and M. Paczuski, Phys. Rev. Lett. 76, 348 (1996).

[15] A condition analogous to our $G^{\prime}(1)=\sum_{i} i p_{i}=1$ is also at the basis of the renormalization group approach to sandpiles of Ref. [4], where it enforces energy balance.

[16] A. L. Stella, C. Tebaldi, and G. Caldarelli, Phys. Rev. E 52, 72 (1995).

[17] C. Tang and P. Bak, J. Stat. Phys. 51, 797 (1988).

[18] S. Lise and A. L. Stella (to be published).

[19] A. B. Harris and T. C. Lubensky, Phys. Rev. B 24, 2656 (1981). 